\title{
On Lorentz Invariant Distributions
}

\author{
Yu. M. Zinoviev \\ Steklov Mathematical Institute, 117333 Moscow, USSR
}

\begin{abstract}
Lorentz invariant tempered distributions with the supports for one-point only in $\bar{V}_{+}^{\mu}$ are described.
\end{abstract}

\section{Introduction}

Lorentz invariant one-point distributions were extensively investigated by P.-D. Methée [1-2]. n-point Lorentz invariant tempered distributions with supports for one-point only in $\bar{V}_{+}^{\mu}$ were studied by K. Hepp [3]. In this case the problem of the description of Lorentz invariant distributions is equivalent to the description of the rotation invariant tempered distributions of $n$ three-vectors. For $n=1,2$ this problem was solved [3]. Rotation invariant distributions and the Lorentz invariant distributions were represented as distributions on the space of the $\mathrm{SO}(3)$-invariants and conformably on the space of the $L_{+}^{\dagger}$-invariants. In trying to generalize Hepp's results to $n>2$ one encounters the difficulty that the space of the $L_{+}^{\uparrow}$-invariants (and the $\mathrm{SO}(3)$-invariants) is an algebraic variety with singularities, on which no reasonable spaces of testing functions have yet been defined [3].

In present paper $\mathrm{SO}(3)$-harmonic analysis on the space $S^{\prime}\left(R^{3}\right)$ is studied. Taking advantage of this analysis it is possible to describe the rotation invariant tempered distributions. As stated above the Lorentz invariant tempered distributions with supports in $\bar{V}_{+}^{\mu} \times R^{4 n}$ were connected with the rotation invariant distributions. Hence we obtain the description of the Lorentz invariant distributions belonging to the space $S^{\prime}\left(\bar{V}_{+}^{\mu} \times R^{4 n}\right)$.

The plan of this paper is as follows: Section 2 contains $\mathrm{SO}(3)$-harmonic analysis on $S^{\prime}\left(R^{3}\right)$; in Section 3 rotation invariant tempered distributions were studied. The Lorentz invariant distributions belonging to $S^{\prime}\left(\bar{V}_{+}^{\mu} \times R^{4 n}\right)$ are under consideration in Section 4. 


\section{Spherical Harmonics}

We shall consider first the spherical harmonics $Y_{l m}(\Theta, \varphi)$, i.e. the eigenvectors of the spherical part of the three-dimensional Lapalace operator. The spherical harmonics $Y_{l m}$ and the associated Legendre functions $P_{l}^{m}$ are related by ([4], p. 24).

$$
Y_{l m}(\Theta, \varphi)=(-1)^{m}\left[\frac{(2 l+1)(l-m) !}{4 \pi(l+m) !}\right]^{1 / 2} P_{l}^{m}(\cos \theta) \exp \operatorname{im} \varphi .
$$

We define the harmonic polynomial $Y_{l m}(x), x \in R^{3}$ as

$$
Y_{l m}(x)=r^{l} Y_{l m}(\Theta, \varphi),
$$

where $r, \Theta, \varphi$ are the spherical coordinates of $x$.

With the distribution $f(x) \in S^{\prime}\left(R^{3}\right)$ and the harmonic polynomial $Y_{l m}(x)$ we relate the linear functional $f_{l m}(t)$ on the space $S\left(\bar{R}_{+}\right)$

$$
\left(f_{l m}(t), \varphi(t)\right)=\left(f(x), Y_{l m}(x) \varphi\left(|x|^{2}\right)\right) .
$$

The function $Y_{l m}(x) \varphi\left(|x|^{2}\right) \in S\left(R^{3}\right)$, and the relation (2.2) is well defined. We call $f_{l m}(t)$ the spherical harmonic of the distribution $f(x)$. It is evident that $f_{l m}(t) \in S^{\prime}\left(\bar{R}_{+}\right)$. For further purposes we need to know how the continuity of $f_{l m}(t)$ depends on $l$. Let us estimate the seminorm $\left\|Y_{l m}(x) \varphi\left(|x|^{2}\right)\right\|_{n, k^{*}}\|\|_{n, k}$ is a usual seminorm on the space $S\left(R^{3}\right)$

$$
\|\varphi(x)\|_{n, k}=\sup _{R^{3}}\left(1+|x|^{2}\right)^{n}\left|\mathscr{D}^{k} \varphi(x)\right|,
$$

where $\mathscr{D}^{k}=\hat{\partial}^{|k|} / \partial x_{1}^{k_{1}} \partial x_{2}^{k_{2}} \partial x_{3}^{k_{3}}$.

Recursion relations for the associated Legendre functions $P_{l}^{m}(x)([4]$, pp. 23-24) are combined for finding the relations for derivatives of $Y_{l m}(x) \varphi\left(|x|^{2}\right)$

$$
\begin{aligned}
& \left(\partial / \partial x_{1}+i \partial / \partial x_{2}\right) Y_{l m}(x) \varphi\left(|x|^{2}\right) \\
& =-2 \alpha_{l m} Y_{l+1, m+1}(x) \varphi^{\prime}\left(|x|^{2}\right)+\alpha_{l-1,-m-1} Y_{l-1, m+1}(x) \Phi_{l}(x) \\
& \partial / \partial x_{3}\left(Y_{l m}(x) \varphi\left(|x|^{2}\right)\right) \\
& =2 \beta_{l+1, m} Y_{l+1, m}(x) \varphi^{\prime}\left(|x|^{2}\right)+\beta_{l m} Y_{l-1, m}(x) \Phi_{l}(x) \\
& \left(\partial / \partial x_{1}-i \partial / \partial x_{2}\right) Y_{l m}(x) \varphi\left(|x|^{2}\right) \\
& =2 \alpha_{l,-m} Y_{l+1, m-1}(x) \varphi^{\prime}\left(|x|^{2}\right)-\alpha_{l-1, m-1} Y_{l-1, m-1}(x) \Phi_{l}(x)
\end{aligned}
$$

where the coefficients

$$
\begin{aligned}
\alpha_{l m} & =[(l+m+1)(l+m+2) /(2 l+1)(2 l+3)]^{1 / 2} \\
\beta_{l m} & =[(l-m)(l+m) /(2 l-1)(2 l+1)]^{1 / 2} \\
\Phi_{l}(x) & =(2 l+1) \varphi\left(|x|^{2}\right)+2|x|^{2} \varphi^{\prime}\left(|x|^{2}\right) .
\end{aligned}
$$

Combining the equations (2.3) and the inequality

$$
\left|Y_{l m}(x)\right|<(2 l+1)^{1 / 2}|x|^{l}
$$


we obtain the estimate

$$
\begin{aligned}
& \left\|Y_{l m}(x) \varphi\left(|x|^{2}\right)\right\|_{n, k} \\
& \leqq C(2 l+1)^{|k|+1} \max _{q \leqq|k|}\|\varphi\|_{n+|k|, q,|k|}^{(l)}
\end{aligned}
$$

where constant $C$ depends on $n$ and $k$, and the seminorm

$$
\|\varphi(t)\|_{n, q, k}^{(l)}=\sup _{\bar{R}_{+}} t^{\frac{1}{2}(l-k)+}(1+t)^{n}\left|\varphi^{(q)}(t)\right|
$$

(here $\left.(l-k)_{+}=\max (l-k, 0)\right)$ is a usual seminorm on the space $S\left(\bar{R}_{+}\right)$.

The application of the inequality (2.4) to the distribution $f(x)$ gives the continuity of the spherical harmonics $f_{l m}(t)$.

Let $S\left(\bar{R}_{+} \times S \hat{S}(3)\right)$ be the space of the sequences $\left\{\varphi_{l m}(t)\right\} \quad(l=0,1, \ldots ; m=$ $-l, \ldots, l)$ of the infinitely differentiable functions $\varphi_{l m}(t)$ in $R_{+}$such that

$$
\max _{l, m}(2 l+1)^{p}\left\|\varphi_{l m}(t)\right\|_{n, q, k}^{(l)}<\infty
$$

for any $p, n, q, k . S\left(\bar{R}_{+} \times \mathrm{SO}(3)\right)$ is locally convex topological vector space with the topology defined by the seminorms that are finite according to (2.5). The inequality (2.4) gives us $\left\{f_{l m}(t)\right\} \in S^{\prime}\left(\bar{R}_{+} \times \mathrm{SO}(3)\right)$.

Let the spherical harmonics $f_{l m}$ be given. We shall consider the problem of the distribution $f(x)$ reconstruction.

Let the function $g(x) \in S\left(R^{3}\right)$. We rewrite it in spherical coordinates: $g(r, \theta, \varphi)=$ $g(r \sin \theta \cos \varphi, r \sin \Theta \sin \varphi, r \cos \Theta)$. We define the function $\tilde{g}_{l m}(r)$ as follows:

$$
\tilde{g}_{l m}(r)=\int_{S^{2}} d \Omega \bar{Y}_{l m}(\Omega) g(r, \Omega)
$$

It is easy to see that $\tilde{g}_{l m}(r) \in S\left(R^{1}\right)$ for any $l, m$. (We allow a negative $r$.) We shall study the properties of $\tilde{g}_{l m}(r)$.

If follows from (2.6) that $\tilde{g}_{l m}(0)=0$ for $l>0$. Let us introduce the differential operators

$$
\begin{aligned}
d_{1} & =(8 \pi / 3)^{1 / 2}\left(\partial / \partial x_{1}-i \partial / \partial x_{2}\right) \\
d_{0} & =(4 \pi / 3)^{1 / 2} \partial / \partial x_{3} \\
d_{-1} & =-(8 \pi / 3)^{1 / 2}\left(\partial / \partial x_{1}+i \partial / \partial x_{2}\right) .
\end{aligned}
$$

We may use the spherical harmonics $Y_{1 m}$ for computing the derivative of $g(r, \Omega)$

$$
(\partial g / \partial r)(r, \Omega)=\sum_{m=-1}^{1} Y_{1 m}(\Omega) d_{m} g(r, \Omega)
$$

In virtue of (2.6) this implies

$$
\left(d^{k} \tilde{g}_{l m} / d r^{k}\right)(0)=\int_{S^{2}} d \Omega \bar{Y}_{l m}(\Omega)\left(\sum_{n=-1}^{1} Y_{1 n}(\Omega) d_{n}\right)^{k} g(0) .
$$

Thus the problem of computing $\tilde{g}_{l m}^{(k)}(0)$ is reduced to that of computing the integrals over the product of the spherical harmonics $Y_{l m}(\Omega)$. These integrals equal zero, if the resultant angular momentum of the addition of the angular momentum $l$ and $k$ angular momenta 1 isn't zero [4]. 


\section{Hence}

$$
\left(d^{k} \tilde{g}_{l m} / d r^{k}\right)(0)=0 \quad k=0, \ldots, l-1 .
$$

Now we show that function $\tilde{g}_{l m}(r)$ has the definite parity. On taking into account the relation (2.6) and

$$
\begin{aligned}
g(-r, \Theta, \varphi) & =g(r, \pi-\Theta, \varphi+\pi) \\
Y_{l m}(\pi-\Theta, \varphi-\pi) & =(-1)^{l} Y_{l m}(\Theta, \varphi)
\end{aligned}
$$

we find

$$
\tilde{g}_{l m}(-r)=(-1)^{l} \tilde{g}_{l m}(r) \text {. }
$$

In view of (2.7) and (2.8) the even function $r^{-l} \tilde{g}_{l m}(r) \in S\left(R^{1}\right)$. Thus there exists the function $g_{l m}(t) \in S\left(\bar{R}_{+}\right)$such that

$$
g_{l m}\left(r^{2}\right)=r^{-l} \tilde{g}_{l m}(r)
$$

(see, for example, [5]). We call $g_{l m}(t)$ the spherical harmonic of the function $g(x)$. We shall prove that for $g(x) \in S\left(R^{3}\right)$ the sequence $\left\{g_{l m}(t)\right\} \in S\left(\bar{R}_{+} \times \mathrm{SO}(3)\right)$. We must show that any seminorm (2.5) is finite on $\left\{g_{l m}(t)\right\}$. First we consider the factor $(2 l+1)^{p}$ in (2.5). The spherical harmonic $Y_{l m}$ is the eigen function of the spherical part $\Delta_{\Omega}$ of the Laplace operator $\Delta$ with the eigenvalue $-l(l+1)([4]$, p. 21$)$. Hence

$$
(2 l+1)^{2 p} g_{l m}(t)=t^{-l / 2} \int_{S^{2}} d \Omega \bar{Y}_{l m}(\Omega)\left(1-4 \Delta_{\Omega}\right)^{p} g\left(t^{1 / 2}, \Omega\right) .
$$

However the function $\left(1-4 \Delta_{\Omega}\right)^{p} g(r, \Omega)$ is the function

$$
g_{(p)}(x)=2^{2 p}\left[\left(\frac{1}{2}+\sum_{i=1}^{3} x_{i} \partial / \partial x_{i}\right)^{2}-|x|^{2} \Delta\right]^{p} g(x)
$$

in spherical coordinates. It is clear that $g_{(p)}(x) \in S\left(R^{3}\right)$. Thus we have

$$
(2 l+1)^{2 p} g_{l m}(t)=g_{(p) l m}(t) .
$$

Let us estimate now the seminorm $\left\|g_{l m}\right\|_{n, q, k}^{(l)}$. First note that

$$
t^{n}\left(d^{k} f / d t^{k}\right)(t)=(t d / d t-(n-1)) t^{n-1}\left(d^{k-1} f / d t^{k-1}\right)(t) \text {. }
$$

This implies for $l \geqq 2 q+k$

$$
\begin{aligned}
& \left\|g_{l m}(t)\right\|_{n, q, k}^{(l)} \\
& \leqq C(2 l+1)^{q} \max _{s \leqq q}\left\|r^{l-k-2 q} g_{l m}\left(r^{2}\right)\right\|_{n+q, s} .
\end{aligned}
$$

We wrote the result in terms of the coordinate $r, r^{2}=t$. The seminorm \|\|$_{n, q}$ is usual seminorm on the space $S\left(R^{1}\right)$

$$
\|\varphi(r)\|_{n, q}=\sup _{R^{1}}\left(1+r^{2}\right)^{n}\left|\varphi^{(q)}(r)\right| .
$$

Similarly for $l<2 q+k$

$$
\begin{aligned}
& \left\|g_{l m}(t)\right\|_{n, q, k}^{(l)} \\
& \leqq C(2 l+1)^{q} \max _{s \leqq \frac{1}{2}(l-k)_{+}}\left\|\left(r^{-1} d / d r\right)^{q-(l-k)_{+} / 2} g_{l m}\left(r^{2}\right)\right\|_{n+q, s} .
\end{aligned}
$$


Using (2.7), (2.11) and the formula for the Taylor remainder terms we have for $l \geqq 2 q+k$

$$
\left\|g_{l m}(t)\right\|_{n, q, k}^{(l)} \leqq C(2 l+1)^{q} \max _{|s| \leqq 3 q+k}\|g(x)\|_{n+q, s}
$$

where constant $C$ depends on $q$ and $k$ only. It is a simple matter to extend this estimate to $l<2 q+k$ by using (2.8) and (2.12). In order to prove $\left\{g_{l m}(t)\right\} \in S\left(\bar{R}_{+} \times\right.$ SÔ(3)) it is sufficient now to use (2.10) and the estimate (2.13) for the function $g_{(p+q)}(x)$. Whence

$$
\begin{aligned}
& \max _{l, m}(2 l+1)^{p}\left\|g_{l m}(t)\right\|_{n, q, k}^{(l)} \\
& \leqq C \max _{|s| \leqq 3 q+k}\left\|g_{(p+q)}(x)\right\|_{n+q, s}
\end{aligned}
$$

and consequently $\left\{g_{l m}(t)\right\} \in S\left(\bar{R}_{+} \times S O \hat{O}(3)\right)$. In particular this implies that for the sequence $\left\{f_{l m}(t)\right\}$ of the spherical harmonics of the distribution $f(x) \in S^{\prime}\left(R^{3}\right)$ the expansion

$$
\sum_{l, m}\left(f_{l m}, g_{l m}\right)
$$

is convergent. We prove it converge to $(f(x), g(x))$. Note that the series

$$
\sum_{l, m} Y_{l m}(x) g_{l m}\left(|x|^{2}\right)
$$

absolutely converge to $g(x)$ at every point [6]. In view of (2.4) and (2.14) it converges to $g(x)$ in the topology of $S\left(R^{3}\right)$. By definition (2.2) of the spherical harmonic $f_{l m}(t)$ of the distribution $f(x)$ we have

$$
(f(x), g(x))=\sum_{l, m}\left(f_{l m}(t), g_{l m}(t)\right) .
$$

Summing up:

\section{Theorem 1. The relation}

$$
(f(x), g(x))=\sum_{l, m}\left(f_{l m}(t), t^{-l / 2} \int_{S^{2}} d \Omega \bar{Y}_{l m}(\Omega) g\left(t^{1 / 2}, \Omega\right)\right)
$$

implies the isomorphism between two topological spaces: $S^{\prime}\left(R^{3}\right)$ and $S^{\prime}\left(\bar{R}_{+} \times \mathrm{SO}(3)\right)$. $S^{\prime}\left(R^{3 n}\right)$.

In Section 3 we consider the rotation invariant distributions from the space

\section{Rotation Invariant Distributions}

Let the distribution $f\left(x_{1}, \ldots, x_{n}\right) \in S^{\prime}\left(R^{3 n}\right)$. Its spherical harmonics $f_{l_{1} m_{1} \ldots l_{n} m_{n}}\left(t_{1}\right.$, $\left.\ldots, t_{n}\right)$ are defined in the similar way to the spherical harmonics of the distribution $f(x) \in S^{\prime}\left(R^{3}\right)$. The sequence $\left\{f_{l_{1} m_{1} \ldots l_{n} m_{n}}\right\}$ belongs to the space $S^{\prime}\left(\left(\bar{R}_{+} \times S O \hat{O}(3)\right)^{n}\right)$. The proof of this is exactly analogous and can be omitted. The distribution $f$ and its spherical harmonics are related by

$$
(f, \varphi)=\sum_{l, m}\left(f_{l m}, \varphi_{l m}\right)
$$


where $\varphi_{l_{1} m_{1} \ldots l_{n} m_{n}}\left(t_{1}, \ldots, t_{n}\right)$ is $n$-dimensional spherical harmonics of the function $\varphi\left(x_{1}, \ldots, x_{n}\right) \in S\left(R^{3 n}\right)$

$$
\varphi_{l m}=t_{1}^{-l_{1 / 2}} \ldots t_{n}^{-l_{n / 2}} \int_{S^{2 \times n}} d^{n} \Omega \bar{Y}_{l_{1} m_{1}}\left(\Omega_{1}\right) \ldots \bar{Y}_{l_{n} m_{n}}\left(\Omega_{n}\right) \varphi .
$$

Let us study how the spherical harmonics of the distribution $f$ vary under the rotation $u \in \mathrm{SO}(3)$. Applying (3.1) for the function $\varphi_{u}=\varphi\left(u x_{1}, \ldots, u x_{n}\right)$ we get

$$
\left(f, \varphi_{u}\right)=\sum_{l, m, k} D_{k_{1} m_{1}}^{\left(l_{1}\right)}(u) \ldots D_{k_{n} m_{n}}^{\left(l_{n}\right)}(u)\left(f_{l m}, \varphi_{l k}\right)
$$

where the matrice $D_{k m}^{(l)}(u)$ represents the rotation $u$ in the $(2 l+1)$-dimensional irreducible representation of the group $\mathrm{SO}(3)$. Unitary matrice $D_{k m}^{(l)}(u)$ continuously depends on $u$. This implies that the series (3.3) is integrable with respect to $d u$, where $d u$ is the invariant normalized Haar measure of $\mathrm{SO}(3)$. In virtue of (3.3) we get for the rotation invariant distribution $f\left(x_{1}, \ldots, x_{n}\right)$

$$
(f, \varphi)=\sum_{l, m, k}\left(f_{l m}, \varphi_{l k}\right) \int_{\operatorname{SO}(3)} d u D_{k_{1} m_{1}}^{\left(l_{1}\right)}(u) \ldots D_{k_{n} m_{n}}^{\left(l_{n}\right)}(u)
$$

Thus the problem of the description any rotation invariant tempered distribution is reduced to that of computing the integral over the product of $n D$ 's. In order to compute this integral we note that the product of two $D$ 's may be expressed in terms of one $D$ function by using the Clebsh-Gordan coefficients $\left(l_{1} m_{1} l_{2} m_{2} \mid l_{1} l_{2} j m\right)([4],(4.3 .1))$ and the integral over the product of three $D$ 's equals the product of two $3-j$ symbols of Wigner $([4],(4.6 .2))$

$$
\left(\begin{array}{ccc}
l_{1} & l_{2} & l_{3} \\
\dot{m}_{1} & m_{2} & m_{3}
\end{array}\right) .
$$

Then

$$
\begin{aligned}
& \int_{\operatorname{SO}(3)} d u D_{k_{1} m_{1}}^{\left(l_{1}\right)}(u) \ldots D_{k_{n} m_{n}}^{\left(l_{n}\right)}(u) \\
& =\sum_{j}\left(\begin{array}{c}
l_{1} \ldots l \\
m_{1} \ldots m_{n}
\end{array}\right)_{j_{1} \ldots j_{n-3}}\left(\begin{array}{l}
l_{1} \ldots l_{n} \\
k_{1} \ldots k_{n}
\end{array}\right)_{j_{1} \ldots j_{n-3}}
\end{aligned}
$$

where the generalized Wigner's symbol ${ }^{1}$

$$
\begin{aligned}
& \left(\begin{array}{lll}
l_{1} & \ldots & l_{n} \\
m_{1} & \ldots & m_{n}
\end{array}\right)_{j_{1} \ldots j_{n-3}} \\
& =\sum_{p}\left(l_{1} m_{1} l_{2} m_{2} \mid l_{1} l_{2} j_{1} p_{1}\right) \ldots\left(\begin{array}{ccc}
j_{n-3} & l_{n-1} & l_{n} \\
p_{n-3} & m_{n-1} & m_{n}
\end{array}\right) .
\end{aligned}
$$

Let us consider the properties of the generalized Wigner's symbols. The properties of the Clebsh-Gordan coefficients may be used to obtain the invariance property and the ortogonal property of the generalized Wigner's symbols stating from the definition (3.6).

\footnotetext{
These generalized Wigner's symbols differ in factors only from those introduced in [7].
} 
We have

$$
\begin{aligned}
& \sum_{k} D_{m_{1} k_{1}}^{\left(l_{1}\right)}(u) \ldots D_{m_{n} k_{n}}^{\left(l_{n}\right)}(u)\left(\begin{array}{l}
l_{1} \ldots l_{n} \\
k_{1} \ldots k_{n}
\end{array}\right)_{j}=\left(\begin{array}{c}
l_{1} \ldots l_{n} \\
m_{1} \ldots m_{n}
\end{array}\right)_{j} \\
& \sum_{m}\left(\begin{array}{c}
l_{1} \ldots l \\
m_{1} \ldots m_{n}
\end{array}\right)_{j}\left(\begin{array}{c}
l_{1} \ldots l_{n} \\
m_{1} \ldots m_{n}
\end{array}\right)_{j^{\prime}} \\
& =\delta_{j_{1} j_{1}} \ldots \delta_{j_{n-3}, j_{n}{ }^{\prime}-3} \delta\left(l_{1} \ldots l_{n}, j_{1} \ldots j_{n-3}\right)
\end{aligned}
$$

where $\delta\left(l_{1} \ldots l_{n}, j_{1} \ldots j_{n-3}\right)=1$ if the natural numbers $l_{1}, \ldots, l_{n} ; j_{1}, \ldots, j_{n-3}$ satisfy the polygonal condition, and is zero otherwise. The polygonal condition for $l_{1}, \ldots, l_{n}$; $j_{1}, \ldots, j_{n-3}$ is as follows: one can construct the polygon such that $l_{1}, \ldots, l_{n}$ correspond to the lengths of sides and $j_{1}, \ldots, j_{n-3}$ correspond to the lengths of the diagonals which get going at the vertex where sides $l_{1}$ and $l_{n}$ intersect. By $P_{n}$ we denote the set of $l_{1}, \ldots, l_{n} ; j_{1}, \ldots, j_{n-3}$ satisfying the polygonal condition.

The invariance property (3.7) and the ortogonal property (3.8) of the generalized Wigner's symbols are analogous to those of the $3-j$ symbols of Wigner $([4]$, (4.3.3), (3.7.8)). In view of (3.8) the generalized Wigner's symbol

$$
\left(\begin{array}{l}
l_{1} \ldots l_{n} \\
m_{1} \ldots m_{n}
\end{array}\right)_{j_{1} \ldots j_{n-3}}
$$

equals zero if $\left(l_{1}, \ldots, l_{n} ; j_{1}, \ldots, j_{n-3}\right) \notin P_{n}$.

Let us return now to the rotation invariant distributions. Let $p=\left(l_{1}, \ldots, l_{n}\right.$; $\left.j_{1}, \ldots, j_{n-3}\right) \in P_{n}$. We define the invariant harmonic of the distribution $f\left(x_{1}, \ldots, x_{n}\right) \in$ $S^{\prime}\left(R^{3 n}\right)$ by

$$
f_{p}=\sum_{m}\left(\begin{array}{c}
l_{1} \ldots l_{n} \\
m_{1} \ldots m_{n}
\end{array}\right)_{j_{1} \ldots j_{n-3}} f_{l m}\left(t_{1}, \ldots, t_{n}\right)
$$

where $f_{l_{1} m_{1} \ldots l_{n} m_{n}}$ are the spherical harmonics of $f$.

Similarly for $\varphi \in S\left(R^{3 n}\right)$

$$
\varphi_{p}=\sum_{m}\left(\begin{array}{c}
l_{1} \ldots l_{n} \\
m_{1} \ldots m_{n}
\end{array}\right)_{j_{1} \ldots j_{n-3}} \varphi_{l m}\left(t_{1}, \ldots, t_{n}\right) .
$$

In virtue of (3.5) the relation (3.4) implies

$$
(f(x), \varphi(x))=\sum_{p \in P_{n}}\left(f_{p}, \varphi_{p}\right)
$$

It follows from the invariance property (3.7) that any term in the sum (3.11) is a rotation invariant distribution from $S^{\prime}\left(R^{3 n}\right)$.

Let us express the invariant harmonics $f_{p}$ and $\varphi_{p}$ in terms of $f$ and $\varphi$. We relate $p=\left(l_{1}, \ldots, l_{n} ; j_{1}, \ldots, j_{n-3}\right) \in P_{n}$ to the invariant spherical harmonic

$$
Y_{p}=\sum_{m}\left(\begin{array}{c}
l_{1} \ldots l \\
m_{1} \ldots m_{n}
\end{array}\right) Y_{j} Y_{l_{1} m_{1}}\left(\Omega_{1}\right) \ldots Y_{l_{n} m_{n}}\left(\Omega_{n}\right) .
$$

It corresponds to the invariant polynomial

$$
Y_{p}\left(x_{1}, \ldots, x_{n}\right)=r_{1}^{l_{1}} \ldots r_{n}^{l_{n}} Y_{p}\left(\Omega_{1}, \ldots, \Omega_{n}\right) \text {. }
$$


By definition of the spherical harmonics of $f$ we get

$$
\left(f_{p}(t), \Psi(t)\right)=\left(f\left(x_{1}, \ldots, x_{n}\right), Y_{p}\left(x_{1}, \ldots, x_{n}\right) \Psi\left(\left|x_{1}\right|^{2}, \ldots,\left|x_{n}\right|^{2}\right)\right)
$$

for every $\Psi \in S\left(\bar{R}_{+}^{n}\right)$.

Similarly

$$
\varphi_{p}=t_{1}^{-l_{1 / 2}} \ldots t_{n}^{-l_{n / 2}} \int_{S^{2 \times n}} d^{n} \Omega \bar{Y}_{p}(\Omega) \varphi\left(t_{1}^{1 / 2}, \Omega_{1}, \ldots, t_{n}^{1 / 2}, \Omega_{n}\right) .
$$

Let $S\left(\bar{R}_{+}^{n} P_{n}\right)$ be the space of the sequences $\left\{\Psi_{p}\right\}\left(p=\left(l_{1}, \ldots, l_{n} ; j_{1}, \ldots, j_{n-3}\right) \in P_{n}\right)$ of the infinitely differentiable functions $\Psi_{p}\left(t_{1}, \ldots, t_{n}\right)$ in $\bar{R}_{+}^{n}$ such that for any $k, m, q, s$.

$$
\max _{p \in P_{n}}\left(2 \sum_{i=1}^{n} l_{i}+1\right)^{k}\left\|\Psi_{p}(t)\right\|_{m, q}(p), s<\infty
$$

where the seminorm $\|\Psi(t)\|_{m, q, s}^{(p)}$ equals

$$
\sup _{\bar{R}_{+}^{n}} t_{1}^{\left(l_{1}-s\right)_{+} / 2} t_{n}^{\left(l_{n}-s\right)_{+} / 2}\left(1+\sum_{i=1}^{n} t_{i}\right)^{m}\left|\mathscr{D}^{q} \Psi(t)\right| .
$$

$S\left(\bar{R}_{+}^{n} P_{n}\right)$ is locally convex topological vector space with the topology defined by the seminorms that are finite according to (3.14).

It is easy to see from the ortogonal property (3.8) that a modulus of any generalized Wigner's symbol is less than one. This implies $\left\{\varphi_{p}\right\} \in S\left(\bar{R}_{+}^{n} P_{n}\right)$ and $\left\{f_{p}\right\} \in S^{\prime}\left(\bar{R}_{+}^{n} P_{n}\right)$ in virtue of (3.10) and (3.9). Inversely any sequence $\left\{f_{p}\right\} \in S^{\prime}\left(\bar{R}_{+}^{n} P_{n}\right)$ defines a rotation invariant distribution from $S^{\prime}\left(R^{3 n}\right)$ by (3.11). More precisely we have

Theorem 2. The relation

$$
(f, \varphi)=\sum_{p \in P_{n^{\prime}}}\left(f_{p}, t_{1}^{-l_{1 / 2}} \ldots t_{n}^{-l_{n / 2}} \int_{S^{2 \times n}} d^{n} \Omega \bar{Y}_{p}(\Omega) \varphi\right)
$$

implies the topological isomorphism between the space of $\mathrm{SO}(3)$-invariant tempered distributions from $S^{\prime}\left(R^{3 n}\right)$ and the space $S^{\prime}\left(\bar{R}_{+}^{n} P_{n}\right)$.

For $n=1$ the set $P_{n}$ is one point $l=0$ (the only connected polygon). Thus the Theorem 2 coincides in this case with the well-known theorem on rotation invariant distributions from $S^{\prime}\left(R^{3}\right)$ [5].

Note that a rotation invariant polynomial $Y_{p}\left(x_{1}, \ldots, x_{n}\right)$ may be represented as a polynomial of the scalar products $\left(x_{i}, x_{j}\right)$ [8]. Thus the sequence of the invariant harmonics $\left\{f_{p}\right\}$ defined by (3.12) is probably a way to define a distribution on the variety of the $\mathrm{SO}(3)$-invariants.

\section{Lorentz Invariant Distributions}

In this section we shall consider the Lorentz invariant tempered distributions with the supports in $\bar{V}_{+}^{\mu} \times R^{4 n}$, where $\bar{V}_{+}^{\mu}=\left\{\left.p\left|p_{0} \geqq V\right| p\right|^{2}+\mu^{2}\right\}$. 
Let ${\overline{R_{+}}}_{+}^{\left(\mu^{2}\right)}$ be the interval $\mu^{2} \leqq t<\infty$. K. Hepp proved that the subspace of $L_{+}^{\uparrow}$ invariant distributions from $S^{\prime}\left(\bar{V}_{+}^{\mu} \times R^{4 n}\right)$ is topologically isomorphic to the subspace of the distributions from $S^{\prime}\left(\bar{R}_{+}^{\left(\mu^{2}\right)} \times R^{n} \times R^{3 n}\right)$ which are $\mathrm{SO}(3)$-invariant in the last variables [3]. We shall describe this isomorphism.

For any $y \in V_{+}$let $L(y)$ be the Lorentz transformation corresponding to the $A(y) \in \operatorname{SL}(2, \mathrm{C})\left(\sigma_{i}\right.$ : Pauli matrices $)$

$$
A(y)=\left[2(y, y)^{1 / 2}\left((y, y)^{1 / 2}+y_{0}\right)\right]^{-1 / 2}\left\{\left((y, y)^{1 / 2}+y_{0}\right) \sigma_{0}+\boldsymbol{y} \boldsymbol{\sigma}\right\} .
$$

It is convenient to define $S\left(\bar{V}_{+}^{\mu} \times R^{4 n}\right)$ as the quotient space of $S\left(V_{+}^{v} \times R^{4 n}\right)$ $(0<\nu<\mu)$ by the subspace of those functions which are zero on $\bar{V}_{+}^{\mu} \times R^{4 n}$. Any function $\varphi \in S\left(V_{+}^{v} \times R^{4 n}\right)$ are related to the function $M \varphi\left(t_{0}, \ldots, t_{n}, x_{1}, \ldots, x_{n}\right) \in$ $S\left(R_{+}^{(v 2)} \times R^{n} \times R^{3 n}\right)$ by

$$
M \varphi=\int d y \delta\left((y, y)-t_{0}\right) \varphi\left(y, L(y)\left(t_{1}, x_{1}\right), \ldots, L(y)\left(t_{n}, x_{n}\right)\right) .
$$

The mapping $M$ implies the above mentioned isomorphism. More precisely for any Lorentz invariant $F \in S^{\prime}\left(\bar{V}_{+}^{\mu} \times R^{4 n}\right)$ there exists a rotation invariant $f(t, x) \in$ $S^{\prime}\left(\bar{R}_{+}^{\left(\mu^{2}\right)} \times R^{n} \times R^{3 n}\right)$ such that

$$
(F, \varphi)=(f(t, x), M \varphi(t, x)) .
$$

Let us use the $\mathrm{SO}(3)$-Fourier transform of $f(t, x)$ in the variables $x_{1}, \ldots, x_{n}$.

The invariant harmonics $f_{p}\left(t_{0}, \ldots, t_{n}, t_{n+1}, \ldots, t_{2 n}\right) \in S^{\prime}\left(\bar{R}_{+}^{\left(\mu^{2}\right)} \times R^{n} \times \bar{R}_{+}^{n}\right)$ are defined in exactly the same way as for the distributions from $S^{\prime}\left(R^{3 n}\right)$. Let the function $\Psi \in S\left(\bar{R}_{+}^{\left(\mu^{2}\right)} \times R^{n} \times \bar{R}_{+}^{n}\right)$. We have

$$
\left(f_{p}, \Psi\right)=\left(f(t, x), Y_{p}(x) \Psi\left(t_{0}, \ldots, t_{n},\left|x_{1}\right|^{2}, \ldots,\left|x_{n}\right|^{2}\right)\right) .
$$

The space $S\left(\bar{R}_{+}^{\left(\mu^{2}\right)} \times R^{n} \times \bar{R}_{+}^{n} P_{n}\right)$ may be defined in the similar way to the space $S\left(\bar{R}_{+}^{n} P_{n}\right)$; for a sequence $\left\{\varphi_{p}\left(t_{0}, \ldots, t_{n}, t_{n+1}, \ldots, t_{2 n}\right)\right\}$ an index $p \in P_{n}$ is related to the variables $t_{n+1}, \ldots, t_{2 n}$ only.

It is clear that the sequence $\left\{f_{p}\right\} \in S^{\prime}\left(\bar{R}_{+}^{\left(\mu^{2}\right)} \times R^{n} \times \bar{R}_{+}^{n} P_{n}\right)$. The distribution $f(t, x)$ and the sequence $\left\{f_{p}\right\}$ of its invariant harmonics are connected by the relation which is analogous to (3.11). Substituting in this relation the invariant harmonics

$$
M_{p} \varphi=t_{n+1}^{-l_{1 / 2}} \ldots t_{2 n}^{-l_{n / 2}} \int_{S^{2 \times n}} d^{n} \Omega \bar{Y}_{p}(\Omega) M \varphi
$$

of the function $M \varphi$ we obtain $(F, \varphi)$ in virtue of (4.3).

In summary:

Proposition 1. The retation

$$
(F, \varphi)=\sum_{p \in P_{n}}\left(f_{p}, M_{p} \varphi\right)
$$

implies the topological isomorphism between the space of $L_{+}^{\uparrow}$-invariant tempered distributions from $S^{\prime}\left(\bar{V}_{+}^{\mu} \times R^{4 n}\right)$ and the space $S^{\prime}\left(\bar{R}_{+}^{\left(\mu^{2}\right)} \times R^{n} \times \bar{R}_{+}^{n} P_{n}\right)$.

It is anticipated that our isomorphism (4.6) can be extended to more general case. 


\section{References}

1. Methée,P.-D.: Comm. math. helv. 28, 225-269 (1954)

2. Gårding, L., Lions, J. L.: Nuovo Cimento, Suppl. 14, 9-66 (1959)

3. Hepp, K.: Helv. Phys. Acta 37, 639-658 (1964)

4. Edmonds, A.R.: Angular Momentum in Quantum Mechanics. Princeton N.J.: Princeton U.P. 1957

5. Schwartz,L.: Séminaire 1954/55, Exposé no. 7

6. Beers, B. L., Dragt, A. J.: J. Math. Phys. 11, 2313-2328 (1970)

7. Jucys, A., Levinsonas, J., Vanagas, V.: Mathematical Apparatus of the Theory of Angular Momentum Vilinius 1960. Translation: New York: Gordon and Breach 1964

8. Weyl, H.: The Classical Groups. Princeton N.J.: Princeton U.P. 1939

Communicated by H. Araki

(Received August 27, 1975) 